\title{
Z s. \\ Left Ventricular Assist Device Implantation to a Child with End Stage Heart Failure In The Novel Coronavirus Pandemic Period
}

\section{Tanıl Özer ( $\nabla$ drtanilozer@gmail.com )}

Health Science University Kartal Kosuyolu High Specialization Training and Research Hospital https://orcid.org/0000-0002-2701-2058

Deniz Günay

Health Science University Kartal Kosuyolu High Specialization Training and Research Hospital

\section{Mehmet Aksüt}

Health Science University Kartal Kosuyolu High Specialization Training and Research Hospital

Özge Altaş

Health Science University Kartal Kosuyolu High Specialization Training and Research Hospital

Emre Gürcü

Health Science University Kartal Kosuyolu High Specialization Training and Research Hospital

Kaan Kırali

Health Science University Kartal Kosuyolu High Specialization Training and Research Hospital

\section{Case Report}

Keywords: COVID-19, coronavirus, LVAD, left ventricular assist device, mechanical circulatory support

Posted Date: May 7th, 2020

DOI: https://doi.org/10.21203/rs.3.rs-27337/v1

License: (c) (i) This work is licensed under a Creative Commons Attribution 4.0 International License. Read Full License 


\section{Abstract}

At the end of the 2019, a novel coronavirus disease (COVID-19) appeared in Wuhan, China and spread rapidly to all over the world. Then, the attentions of almost all health care providers have shifted to this health emergency situation which is identified as pandemic. The novel coronavirus pandemic has impacted the physicians at the all field of medicine not to perform their routine treatment practice. Since it is not clear when this situation will end, there is still debate about how to manage the treatment strategies for the patients who have end stage heart failure such another life threatening illness. The aim of this case presentation is to create a consideration about timing of left ventricular assist device implantations during novel coronavirus pandemic period.

\section{Introduction}

At the end of the 2019, a novel coronavirus disease (COVID-19) appeared in Wuhan, China and spread rapidly to all over the world. This outbreak disease which was caused by the SARS coronavirus 2 (SARSCoV-2) is associated with atypical pneumonia and progress to acute respiratory failure (1). After the World Health Organization (WHO) has declared this disease as a pandemic, health organizations of almost all countries focused on it and other deferrable interventions were postponed. The routine health care giving practices were changed and hospitalization criteria for other disease were narrowed. On the other hand, if we consider about the patients with heart failure who mostly carry the risk of admission to hospital with similar symptoms with COVID-19 like fatigue, dyspnea, gastrointestinal complaints etc., the approaches for these patients might be different (2). While it is strongly recommended by health professionals to the public that to delay admission to hospitals as possible as can be, it will be difficult for the patients with heart failure to comply with this warnings (3). In recent years, before this novel pandemic period, the practice to LVAD implantation have been inclined to be performed to the patients in relatively better condition in many clinics (4). In addition to contribution of long term mechanical circulatory support (MCS) systems to the patients' quality of life, the patients who are followed with medical therapy may be faced to rapid deterioration and then the success of intervention may be negatively affected (5). Thus, since it is not clear when this situation will end, it may be considered that not to postpone of implantation of long term left ventricular assist devices (LVAD) to the patients particularly in INTERMACS (Interagency Registry for Mechanically Assisted Circulatory Support) 4 and 5. With this case presentation we aimed to create a consideration about timing of left ventricular assist device implantations during novel coronavirus pandemic period.

\section{Case Presentation}

Informed consent to publish the data was taken from the parents. An 11-year-old female patient admitted to the pediatric emergency department (ED) of another hospital with epigastric pain and dyspnoea without fever. She had not growth retardancy background and any risk factors for cardiomyopathy (CMP) but had a history of upper respiratory tract infection which recovered without medical treatment 10 days before. The patient seemed to be restless, pale and oriented at the first examination. Jugular venous 
distention was prominent. Physical examinations revealed tachycardia $(130 / \mathrm{min})$ and tachypnea $(27 / \mathrm{min})$. Patient was normotensive $(100 / 60 \mathrm{mmHg})$. On pulse oximeter, oxygen saturation was $99 \%$. Hepatomegaly and systolic ejection murmur $(2 / 6)$ was detected. Blood biochemistry revealed elevated ALT (343 U/L), LDH (603 U/L) and Troponin T (2,5 ng/ml) levels. White blood cell, red blood cell and platelet counts were normal. Heart silhouette seemed to be increased on chest X-Ray. Thoracic ultrasonography revealed bilateral pleural effusion. Reduced left ventricular ejection fraction (15-20\%) with dilated ventricles detected with echocardiography. Patient was diagnosed as dilated cardiomyopathy with myocarditis background. None of the possible pathogens were detected in blood and swab samples. Nasopharyngeal and oropharyngeal swabs were taken for RT-PCR analysis of COVID19 at two different times and both of them resulted as negative. Medical treatments applied to the patient were methylprednisolone ( $2 \mathrm{mg} / \mathrm{kg} /$ day iv.), enalapril ( $0,1 \mathrm{mg} / \mathrm{kg} /$ day po.), carnitine (2 gr/ day po.), coenzyme-Q (100mg/day po.) and furosemide $(0,1 \mathrm{mg} / \mathrm{kg} / \mathrm{h}$ iv.). There was no sign of recovery at the end of the 14th day of the therapy and referred our hospital for the evaluation of further therapies.

Patient was at INTERMACS level 4 at the admission of our hospital. Patients was immediately included to transplantation list but unfortunately it was known that the expectancy of heart transplantation was very low due to current pandemic situation. Echocardiography revealed left ventricular dilatation (LVEDD:7 cm), impaired left ventricular systolic function (LVEF:23\% with biplane Simpson's method), moderate right ventricular dysfunction (TAPSE: $10 \mathrm{~mm}$ ), moderate mitral and tricuspid valve regurgitation and none of the congenital abnormalities. Cranial CT scan is completely normal. Thorax CT images were compatible with pulmonary edema and pleural effusion. Abdominal USG revealed hepatomegaly (craniocaudal diameter is $154 \mathrm{~mm}$ ) with minimal ascites. ALT (399 U/L), LDH(602 U/L), Troponin-T(1,7), total bilirubin $(1,23 \mathrm{mg} / \mathrm{dl})$ levels were elevated in Blood chemistry analyses. Creatinine $(0,42 \mathrm{mg} / \mathrm{dl})$ and albumin $(33 \mathrm{~g} / \mathrm{L})$ levels were found decreased. INR was 1,46 . Neutrophilia $\left(16,710^{\wedge} 3 / \mu \mathrm{L}\right)$ and leukocytosis $\left(19,810^{\wedge} 3 / \mu \mathrm{L}\right)$ were detected in complete blood count and considered to be related with steroid therapy.

Anemia and thrombocytopenia were not detected. During the follow-up there were only two treatment strategy discussed for this girl, due to low probability for transplantation. They were LVAD implantation in elective status without delaying or continue medical treatment during pandemic period. In a few day on follow up, hypotension occurred and milrinone $(0,5 \mathrm{mcg} / \mathrm{kg} / \mathrm{min}$.) and noradrenaline $(0,2 \mathrm{mcg} / \mathrm{kg} / \mathrm{min})$ therapies were started. The patient was stable under low-dosage of inotropic support. IVIG therapy (1 $\mathrm{g} / \mathrm{kg} /$ day, 2 day) was added to treatment. But there was no clinical and laboratory improvement after that. After two days, patient's condition deteriorated rapidly and santral ECMO was implanted as a transient mechanical circulatory support (MCS) under medical cardiopulmonary resuscitation (mCPR). Biatrial inflow and ascendant aortic outflow implantations were chosen for both right and left atrial decompression. Brain and organ functions were determined as preserved. After a few days thrombocytopenia and anemia developed and then replaced. After the day 7 patient under ECMO and higher dose inotropic support (INTERMACS 2) echocardiographic evaluation showed severe bi-ventricular disfunction. Left ventricular assist device therapy was considered as long term MCS to provide patient mobility. Surgery was planned with the informed consent of the parents. HeartWare HVAD (Medtronic, 
Minneapolis, MN) was implanted as LVAD with conventional full sternotomy. Severe right ventricular failure occurred early after the implantation and temporary right ventricular circulatory support implanted from right atrium to pulmonary artery. Patient was stable under low dose of inotropic and temporary right ventricular circulatory support $(1 \mathrm{l} / \mathrm{min} / \mathrm{m} 2)$ while LVAD flow was $1.7 \mathrm{l} / \mathrm{min}$. The follow up has been continuing for 22 days and patient has not been discharged from ICU yet during this presentation prepared.

\section{Discussion}

The novel coronavirus outbreak first occurred in Wuhan, Chine in December 2019 and spread rapidly to all over the world. The health providing systems have changed and focused on COVID-19 disease since the first case was declared at March 11, 2020 in Turkey, like have been in almost all countries. In general meaning, this case presentation could be seen as it didn't has any novel treatment strategies or any technical attributes. But in our knowledge, LVAD implantation in novel coronavirus pandemic period was not declared in any clinic and thus, it seems LVAD implantations to the patients whether COVID positive or not, were postponed at most of clinics. In our clinic after first case was declared at March 11 in Turkey, our approaches to the patients who were planned to LVAD implantation were mostly favor to postpone. However, with the advancing time, our thought turned to that this group of patients may be more risky in this uncertain process. In normal periods, most of physicians who interested in heart failure treatments recommend that implantation of LVAD to the patients in the INTERMACS 4 or 5 levels, rather than to the same patients after worsening to the lower INTERMACS levels $(4,5)$. The implantation strategies to the children were substantially different previously than adult with same conditions. However, lately the strategies turned to similar with adults due to the development of relatively smaller sized devices (6). In fact, if our present case who was an 11-year-old girl with heart failure related to myocarditis would have happened in normal period, we could plan to implant a LVAD earlier, but due to confusion of this unfamiliar pandemic period we could not achieve consensus between the clinics of cardiovascular surgery, pediatric cardiology and anesthesiology. Eventually we decided to extend the medical treatment process as much as possible and avoid surgical procedures.

Even, our hospital was not yet accepted as a pandemic hospital. In literature some controversial thoughts have been present and published about intravenous immunoglobulin (IVIG) therapy $(7,8)$. As it was mentioned above our initial approach was to continue with medical therapy which included IVIG during the patient was stable with IV diuretic and low dose inotrope. But we could not get a benefit from this therapy.

In general public health practices, due to the virus spreading rapidly in pandemic situations, most of cases except those requiring urgency are postponed with prediction of overwhelming and lack of bed in hospitals (9). It is also known that surgical and medical treatments for chronic heart failure do not exist among cardiac emergencies which can be assumed as exceptional condition (10). However, it is also stated that patients with chronic heart failure can frequently apply to emergency departments, despite maximal medical treatment, and very few are sent back to home (11). Considering the burden of 
hospitals during pandemic periods, attempts to reduce the frequency of hospital admissions of these patients can be considered not only for individual health but also for public health. In the present case, initially we didn't meet this thought, and we were of the opinion that extending the duration of medical treatment to as long as could been would have been the best option. But, the condition of our patient rapidly decreased from INTERMACS 4 to INTERMACS 2, and the healing process after LVAD implantation has not yet been satisfactory, similar with the comparative studies (5).

There are limited publications on elective surgical procedures during the coronavirus pandemic period. In the study of Lei et al., mortality rates of surgeries applied to patients in the incubation period were noticeably high. This rate was seen to be much higher, especially in the high-risk patient group (12). Left ventricular assist device implantations that if were considered as high-risk surgery, even these patients have been operated at the incubation period, would be at high risk. The cultures which were taken from our patient 2 times consecutively were resulted negative, and there were no radiological findings suggesting COVID-19 disease. But, the lack of experience in both our clinic and the literature restricted us to choose the most appropriate approach related to such processes.

It is known that individuals with any chronic disease constituted the most risky population during the coronavirus pandemic period (2). As with all patients with chronic diseases such as cancer, any implementations that are applied in order to minimize the admission to hospital and even remote monitoring of patients gain importance (13). Furthermore, the preference of the applications that will shorten the hospitalization period during surgical intervention may also be useful (14). On the other hand, self - measurement of International Normalized Ratio (INR) which has been the most frequently followed laboratory parameter in LVAD patients, could also contribute to reducing hospital admissions (15).

In conclusion, performing LVAD implantation to the patients in INTERMACS 4 or 5 as soon as possible may be a more effective approach, rather than delaying, in coronavirus pandemic process, in which we do not have enough information about the process yet. We believe that this case report may also give insight into comparative studies that will enable us to make more accurate decisions.

\section{Declarations}

We declare there is no conflict of interest at any stage of this study

\section{References}

1. Zhu N, Zhang D, Wang W, et al. A novel coronavirus from patients with pneumonia inchina.N Engl J Med. 2019; 382 (2020727-33).

2. Wang D, Hu B, Hu C, et al. Clinical characteristics of 138 hospitalized patients with 2019 novel coronavirus-infected pneumonia in Wuhan, China. JAMA 2020 February 7 (Epub ahead of print).

3. https://www.who.int/emergencies/diseases/novel-coronavirus-2019/advice-for-public. 
4. Stewart GC, Kittleson MM, Patel PC, et al. INTERMACS (Interagency Registry for Mechanically Assisted Circulatory Support) Profiling Identifies Ambulatory Patients at High Risk on Medical Therapy After Hospitalizations for Heart Failure. Circulation Heart failure 2016;9.

5. Shah KB, Starling RC, Rogers JG, et.al. ROADMAP Investigators. Left ventricular assist devices versus medical management in ambulatory heart failure patients: an analysis of INTERMACS Profiles 4 and 5 to 7 from the ROADMAP J Heart Lung Transplant. 2018; 37:706-714.

6. Miera O, Potapov EV, Redlin M, et al. First experiences with the HeartWare ventricular assist system in children. Ann. Thorac. Surg. 2011;91(4):1256-1260.

7. Butts RJ, Boyle GJ, Deshpande SR, et al. Characteristics of Clinically Diagnosed Pediatric Myocarditis in a Contemporary Multi-Center Cohort. Pediatr Cardiol 38, 1175-1182 (2017). https://doi.org/10.1007/s00246-017-1638-1.

8. Huang $X$, Sun $Y$, Su G, et al. Intravenous immunoglobulin therapy for acute myocarditis in children and adults. Int. Heart J. 2019 Mar 20; 60: pp. 359-365.

9. Levin PJ, Gebbie EN, Qureshi K. Can the health-care system meet the challenge of pandemic flu? Planning, ethical, and workforce considerations. Public Health Rep. 2007;122:573-578.

10. Chang YJ, Chang SL, Chong E, et al. Cardiovascular Emergencies. Biomed Research 2017; 1-2.

11. Shafazand M, Patel H, Ekman I. Patients with worsening chronic heart failure who present to a hospital emergency department require hospital care. BMC Res Notes 2012; 5:

12. Lei S, Jiang F, Su W, et al. Clinical characteristics and outcomes of patients undergoing surgeries during the incubation period of COVID-19 infection [published online ahead of print, $2020 \mathrm{Apr} 5$ ]. EClinicalMedicine. 2020;100331.

13. Al-Shamsi HO, Alhazzani W, Alhuraiji A, et al. A Practical Approach to the Management of Cancer Patients During the Novel Coronavirus Disease 2019 (COVID-19) Pandemic: An International Collaborative Group. Oncologist. 2020 Apr 3. doi: 10.1634/theoncologist. 2020-0213. [Epub ahead of print].

14. Ozer T, Gunay D, Hancer H, et al. Transition from Conventional Technique to Less Invasive Approach in Left Ventricular Assist Device Implantations. ASAIO J. 2020 Jan 21. doi: 10.1097/ 0000000000001123. [Epub ahead of print].

15. Heneghan C, Ward A, Perera R, et al. Self-monitoring of oral anticoagulation: systematic review and meta-analysis of individual patient data. Lancet 2012;379:322-334. 